Yael Hacohen, MRCPCH

Rahul Singh, MRCPCH

Vhari Forsyth, MRCPCH

Michael Absoud,

MRCPCH, PhD

Ming Lim, MRCPCH,

$\mathrm{PhD}$

Correspondence to

Dr. Lim:

ming.lim@gstt.nhs.uk

\title{
CSF albumin and immunoglobulin analyses in childhood neurologic disorders OPEN
}

\section{ABSTRACT}

Objective: To evaluate the utility of qualitative and quantitative analyses of CSF immunoglobulins as part of the diagnostic workup of CNS inflammatory conditions.

Methods: One hundred eighty-nine children who underwent CSF investigation for their neurologic condition had CSF and serum testing to (1) qualitatively identify oligoclonal band (OCB) patterns and (2) quantitatively measure the immunoglobulin (lg) G index and albumin quotient (QAlb). Case notes were retrospectively reviewed and patients were grouped according to whether their primary diagnosis was due to an inflammatory $(n=104)$ or noninflammatory $(n=85)$ etiology.

Results: CSF-restricted OCBs were found in 20/104 (19\%) of the inflammatory group compared with $4 / 85(5 \%)$ of the noninflammatory group ( $p=0.0036)$. Mirrored OCBs were found in 13/104 (12.5\%) of the inflammatory group compared with 5/85 (6\%) of the noninflammatory group ( $p=0.14)$. IgG index and $Q_{\text {Alb }}$ were significantly higher in patients with an inflammatory etiology. However, a raised lgG index (>0.85) and $Q_{\text {Alb }}(>0.049)$ were seen in both groups, with $Q_{\text {Alb }}$ abnormalities seen more frequently in the inflammatory group ( $p=0.0028)$.

Conclusions: Both methods were informative in identifying inflammatory mechanisms. Abnormalities were more commonly, but not exclusively, seen in primary inflammatory conditions. The qualitative and quantitative evaluation collectively revealed additional positive results than when done in isolation. Neurol Neuroimmunol Neuroinflammation 2014;1:e10; doi: 10.1212/NXI.0000000000000010

\section{GLOSSARY}

ICD-10 = International Classification of Diseases, tenth revision; $\mathbf{I g}=$ immunoglobulin; $\mathbf{M S}=$ multiple sclerosis; $\mathbf{O C B}=$ oligoclonal band; QAlb = albumin quotient.

CSF analysis of immunoglobulins (Igs; often IgG) includes a qualitative analysis of concurrent sera and CSF to identify the 5 characteristic oligoclonal band (OCB) patterns. ${ }^{1}$ Type 1 is a normal pattern where no bands are identified. A type 2 pattern indicates intrathecal synthesis, where bands are seen only in the CSF. When the pattern of bands seen is identical in both sera and CSF, a "mirrored" type 4 pattern is recorded, demonstrating that the IgG has passively diffused into the CNS. Sometimes the pattern identified has identical shared bands but additional CSF-specific bands, a type 3 pattern. On rare occasions, a type 5 pattern is seen, in which a monoclonal $\operatorname{IgG}$ band is identified in serum and CSF (detailed description provided in reference 1). In addition, the CSF and sera can be quantitatively analyzed by measuring the albumin quotient $\left(\mathrm{Q}_{\mathrm{Alb}}=\mathrm{Alb}_{\mathrm{CSF}} / \mathrm{Alb}_{\mathrm{SERUM}}\right)$ and $\mathrm{IgG}$ index $(\mathrm{IgG}$ Index $\left.=\mathrm{IgG}_{\mathrm{CSF}} / \mathrm{IgG}_{\text {SERUM }}\right) /\left(\mathrm{Alb}_{\mathrm{CSF}} / \mathrm{Alb}_{\mathrm{SERUM}}\right)$ to evaluate blood-brain barrier dysfunction. ${ }^{1}$

The quantitative analysis of sera and CSF has little added value to the qualitative analysis of bands in the diagnosis of multiple sclerosis (MS), ${ }^{1}$ although it is less clear whether this is the case across the range of neurologic disorders. Two studies from more than 2 decades ago have studied qualitative and quantitative CSF analysis in a range of neurologic conditions. The first study highlighted the additional value of testing serum and CSF together and identified identical bands in the serum in $50 \%(56 / 112)$ of the patients, suggesting a systemic immune response. ${ }^{2}$ The second study, which

From Children's Neuroscience, Evelina London Children's Hospital at Guy's \& St Thomas' NHS Trust, King's Health Partners AHSC, Lambeth Palace Road, London.

Go to Neurology.org/nn for full disclosures. Funding information and disclosures deemed relevant by the authors, if any, are provided at the end of the article. The Article Processing Charge was paid by the authors.

This is an open access article distributed under the terms of the Creative Commons Attribution-Noncommercial No Derivative 3.0 License, which permits downloading and sharing the work provided it is properly cited. The work cannot be changed in any way or used commercially. 
Table 1 ICD-10 inflammatory diseases of the central and peripheral nervous system and the qualitative (oligoclonal band pattern) and quantitative (IgG index and $\left.Q_{A l b}\right)$ analysis

\begin{tabular}{|c|c|c|c|c|c|c|c|}
\hline & ICD-10 & $\begin{array}{l}\text { Type 1, } \\
\text { no OCB }\end{array}$ & $\begin{array}{l}\text { Type 2, } \\
\text { intrathecal }\end{array}$ & $\begin{array}{l}\text { Type 3, mirrored } \\
\text { and intrathecal }\end{array}$ & $\begin{array}{l}\text { Type 4, } \\
\text { mirrored }\end{array}$ & $\begin{array}{l}\text { Raised IgG index } \\
(>0.85)\end{array}$ & $\begin{array}{l}\text { Raised } Q_{\text {Alb }} \\
\left(>4.9 \times 10^{-3}\right)\end{array}$ \\
\hline All inflammatory $(n=104)$ & & 72 (69) & $10(10)$ & $10(10)$ & $13(13)$ & $21(20)$ & $27(26)$ \\
\hline $\begin{array}{l}\text { Non-MS relapsing demyelination } \\
(n=5)\end{array}$ & G37.9 & $3(60)$ & $2(40)$ & 0 & 0 & $1(20)$ & $2(40)$ \\
\hline $\operatorname{MS}(n=3)^{b}$ & G35 & 0 & $3(100)$ & 0 & 0 & $2(67)$ & 0 \\
\hline $\begin{array}{l}\text { Antibody-mediated encephalitis } \\
(n=5)\end{array}$ & G05.8 & $5(100)$ & 0 & 0 & 0 & 0 & 0 \\
\hline Encephalopathies (immune) $(n=15)$ & G05.8 & $12(80)$ & 0 & $2(13)$ & $1(7)$ & $2(13)$ & $4(27)$ \\
\hline CNS vasculitis $(n=3)$ & G08; 167.6 & 1 (33) & 1 (33) & 1 (33) & 0 & 0 & 1 (33) \\
\hline Peripheral demyelination $(n=14)$ & G61 & $10(71)$ & 0 & 0 & $4(29)$ & $4(29)$ & $8(58)$ \\
\hline Uveitis ( $\mathrm{n}=3$ ) & $\mathrm{H} 2 \mathrm{O}$ & $3(100)$ & 0 & 0 & 0 & 0 & 0 \\
\hline All noninflammatory $(\mathrm{n}=\mathbf{8 5})$ & & $76(89)$ & $3(4)$ & $1(1)$ & $5(6)$ & $14(16)$ & $7(8)$ \\
\hline Seizure disorder $(n=22)$ & $\mathrm{G} 40$ & $19(87)$ & $1(5)$ & 0 & $2(9)$ & $2(9)$ & $2(9)$ \\
\hline Neuroregression $(n=13)$ & G31 & $11(85)$ & 0 & 0 & 2 (15) & $4(31)$ & $2(15)$ \\
\hline Neurometabolic disorder $(n=11)$ & E70-90 & $10(91)$ & 1 (9) & 0 & 0 & 4 (36) & $1(9)$ \\
\hline Headaches ( $n=8 ; 6 \Perp H, 2$ migraine) & G43; G93.2; R51 & $8(100)$ & 0 & 0 & 0 & 0 & 0 \\
\hline Developmental delay $(n=6)$ & F70-79 & $5(83)$ & $1(17)$ & 0 & 0 & 0 & 0 \\
\hline Movement disorder $(n=6)$ & G20-26 & $6(100)$ & 0 & 0 & 0 & 0 & 0 \\
\hline Vascular $(n=2)$ & 163 & $2(100)$ & 0 & 0 & 0 & $1(50)$ & 0 \\
\hline Peripheral neuropathy $(n=1)$ & G62 & $1(100)$ & 0 & 0 & 0 & 0 & 0 \\
\hline
\end{tabular}

Abbreviations: ADS = acquired demyelinating syndromes; HSV = herpes simplex virus; ICD-10 = International Classification of Diseases, tenth revision; $\mathrm{lg}=$ immunoglobulin; IIH = idiopathic intracranial hypertension; $\mathrm{MS}=$ multiple sclerosis; $\mathrm{OCB}=$ oligoclonal band; PRES = posterior reversible encephalopathy syndrome; $\mathrm{Q}_{\mathrm{Alb}}=$ albumin quotient; $\mathrm{VZV}$ = varicella-zoster virus.

Data are $\mathrm{n}(\%)$, except for the ICD-10 column.

a Demyelinating phenotypes were classified based on International Pediatric MS Study Group criteria ${ }^{7}$ into monophasic ADS (15 acute disseminated encephalomyelitis, 2 optic neuritis, 7 transverse myelitis, and 4 other clinically isolated syndrome) and relapsing phenotypes. One child with non-MS relapsing demyelination was diagnosed with neuromyelitis optica.

${ }^{\mathrm{b}}$ Twelve children were diagnosed with MS at our center during the study period. Four had CSF analysis done at the referring institution, 2 had a firm clinical diagnosis (and had not had CSF studies), and 3 were between the ages of 16 and 18 years and hence were not included.

${ }^{c}$ All 11 patients with a diagnosis of CNS infection had the relevant serum and CSF investigations to confirm the diagnosis; of these, 3 patients had bacterial meningitis/myelitis, 4 had CSF PCR-proven viral encephalitis (3 HSV, 1 VZV), 2 had clinical syndrome associated with rising viral titers (1 H1N1, 1 influenza A), and 2 had presumed viral encephalitis.

${ }^{d}$ Four patients with 7 th nerve palsy, 2 with 6 th, 1 with 3 rd, and 1 with multiple cranial neuropathies.

was the first pediatric study, was very informative but included only 33 children (out of the 161 studied) with inflammatory conditions. ${ }^{3}$ A contemporary Australian study ${ }^{4}$ reported the diagnostic value of qualitative CSF IgG analysis in a range of childhood-onset neurologic diseases. Therefore, the aim of this study was to evaluate the utility of the qualitative and quantitative evaluation of the CSF when investigating children with CNS inflammatory conditions. 
METHODS Between 2007 and 2012, a total of 189 consecutive children (ages 3 months to 16 years, median age 8 years) who underwent CSF investigation for their suspected inflammatory neurologic condition at a tertiary pediatric neurology center had CSF and serum testing to (1) qualitatively identify OCB patterns type $1-5$ by isoelectric focusing on agarose gels, followed by immunoblotting as previously described ${ }^{1}$; and (2) quantitatively measure the IgG index and $\mathrm{Q}_{\mathrm{Alb}}$ as previously reported. ${ }^{5,6}$ If multiple samples were tested ( $\mathrm{n}=$ 11), results from the first sample were reported and used in analysis. CSF IgG analysis was not used in designating the classification of the patients' diagnosis. In our institution, the investigations protocol for a child with a suspected inflammatory disorder includes both qualitative and quantitative CSF Ig analysis.

Patient case notes were retrospectively reviewed (Y.H., R.S., V.F.) and patients were classified (Y.H., M.A., M.L.) using the ICD-10 as having inflammatory diseases of the central and peripheral nervous system $(\mathrm{n}=104)$ or noninflammatory etiology $(\mathrm{n}=85)$. Demyelinating phenotypes were classified based on the International Pediatric MS Study Group criteria ${ }^{7}$ into monophasic acquired demyelinating syndromes (acute disseminated encephalomyelitis, optic neuritis, transverse myelitis, or other clinically isolated syndrome) and relapsing phenotypes. Patients with autoimmune encephalopathies were subdivided into those with a known neuronal autoantibody and those with probable clinical diagnosis, as previously described. ${ }^{8}$ All patients with a diagnosis of CNS infection had the relevant serum and CSF investigations to confirm the diagnosis. Descriptive statistics were used to summarize the key components of patient data. Fisher exact (2-tailed) and Mann-Whitney tests were used for comparison of clinical details and investigatory findings in the different groups.

Standard protocol approvals, registrations, and patient consents. Institutional approval was obtained to perform this evaluation as a clinic audit (Ref 1782).

RESULTS The qualitative and quantitative CSF and sera IgG abnormalities and $\mathrm{Q}_{\mathrm{Alb}}$ are reported in table 1, stratified by presence of a primary inflammatory pathology. Patients with an inflammatory condition were significantly more likely to have abnormal results $(51 / 104$ [49\%] compared to 26/85 [31\%]; $p=0.012$, Fisher exact test). Abnormal qualitative results were identified in $33 / 104(32 \%)$ and $9 / 85(11 \%)$, respectively ( $p=$ 0.0007 , Fisher exact test). Intrathecal CSF OCBs (types $2+3$ ) were found in 20/104 (19\%) (type 2, $\mathrm{n}=10$; type $3, \mathrm{n}=10)$ of the inflammatory group compared with $4 / 85(5 \%)($ type $2, \mathrm{n}=3$; type $3, \mathrm{n}=$ 1) of the noninflammatory group ( $p=0.0036$, Fisher exact test). Mirrored OCBs (type 4) were found in $13 / 104(12.5 \%)$ of the inflammatory group compared with $5 / 85(6 \%)$ of the noninflammatory group $(p=$ 0.14 , Fisher exact test). The quantitative analyses are reported in table 2 and illustrated in the figure. The absolute measures of IgG index and $\mathrm{Q}_{\text {Alb }}$ are significantly higher in patients with an inflammatory etiology (table 2, $p=0.048$ and $p=0.0098$, respectively; Mann-Whitney test). Raised IgG index $(>0.85)$ was seen in both groups (table 2), whereas $\mathrm{Q}_{\mathrm{Alb}}(>0.049)$ abnormalities were seen more frequently in the inflammatory group ( $p=0.0028$, Fisher exact test). The qualitative and quantitative evaluation collectively revealed additional positive results than when done in isolation (figure 1, A and B), Overall, the likelihood ratio of a positive test (type 2-4 pattern vs type 1 pattern) to help differentiate inflammatory from noninflammatory causes was 3.1 (95\% confidence interval 1.6-6.0).

DISCUSSION In this study of 189 children, both quantitative and qualitative analyses were informative in identifying inflammatory mechanisms. Intrathecal OCBs were present in $32 \%$ of patients with inflammatory CNS disorders. OCBs were also identified in the noninflammatory group, albeit at a lower frequency (11\%). Four patients had type 2 and 3 OCBs in the noninflammatory group (table 1). All these patients did not have features atypical to their diagnosis. These data are similar to those reported by Sinclair et al., ${ }^{4}$ who identified intrathecal OCBs in 11/58 (19\%) of patients with inflammatory conditions and in none of 142 patients with noninflammatory conditions. In contrast, we additionally identified 11 patients (predominantly in the inflammatory group) with a combination mirrored and intrathecal OCB pattern (type 3).

To date, our study is the first contemporary cohort to evaluate the utility of the quantitative analysis in a range

Table 2 Comparison of quantitative results between the inflammatory and noninflammatory groups additionally subdivided into patients with normal and abnormal oligoclonal band pattern

\begin{tabular}{|c|c|c|c|}
\hline & Inflammatory ( $n=105$ ) & Noninflammatory $(n=85)$ & p Value \\
\hline Age, y & $8(1-16)$ & $7(0.4-16)$ & 0.022 \\
\hline IgG index $(n=77,54)$ & $0.71(0.11-7.16)$ & $0.62(0.13-4.94)$ & 0.0484 \\
\hline $\lg G$ index $>0.85$ & $21 / 77(27)$ & $14 / 54(26)$ & NS \\
\hline$Q_{\text {Alb }}>4.9 \times 10^{-3}(n=83,63)$ & $0.0033(0.00035-0.046)$ & $0.0026(0.00032-0.03)$ & 0.0098 \\
\hline $\mathrm{Q}_{\mathrm{Alb}}>4.9 \times 10^{-3}$ & 27/83 (33) & $7 / 63(11)$ & 0.0028 \\
\hline Isolated OCB only (type $2+3$ ) (normal IgG index, $Q_{\text {Alb) }}$ & $4 / 20(20)$ & $2 / 9(22)$ & NS \\
\hline Isolated OCB only (type 4) (normal IgG index, $Q_{\text {Alb) }}$ & $4 / 13(31)$ & $1 / 5(20)$ & NS \\
\hline
\end{tabular}

Abbreviations: Ig = immunoglobulin; NS = nonsignificant; OCB = oligoclonal band; $\mathrm{Q}_{\mathrm{Alb}}=$ albumin quotient. Data are median (range) or $\mathrm{n} / \mathrm{N}(\%)$. 
A

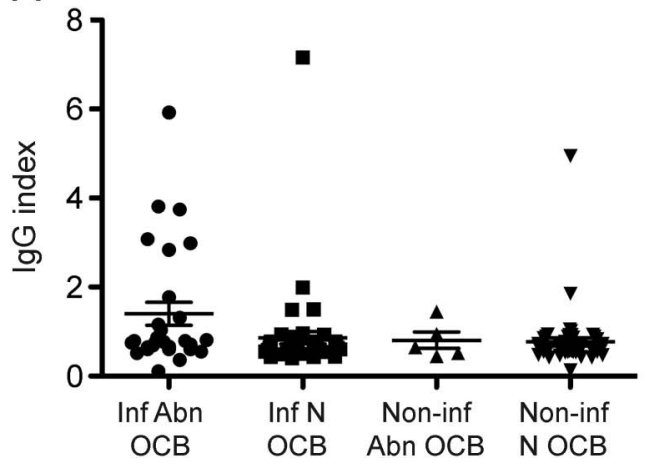

B

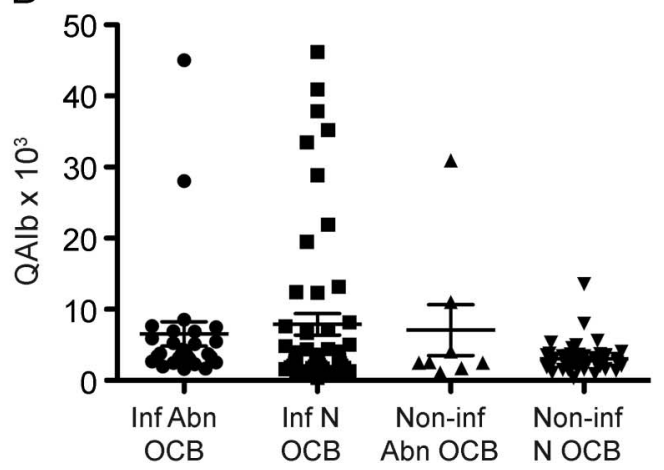

Immunoglobulin (Ig) G index and albumin quotient $\left(Q_{\text {Alb }}\right)$ abnormalities were seen in all 4 groups. Vertical bars represent mean and SD. Abnormal quantitative results (both IgG index and $\mathrm{Q}_{\text {Alb }}$ ) were seen in patients with normal oligoclonal band (OCB) pattern. Abn = abnormal; inf = inflammatory; $\mathrm{N}=$ normal; non-inf = non-inflammatory.

of childhood neurologic disorders. Raised IgG index in isolation was not found in significantly higher incidence in the inflammatory group and did not have specificity for a specific diagnosis or disease group, whereas raised $Q_{\text {Alb }}$ was seen more frequently in patients with inflammatory conditions. Nevertheless, IgG index and QAlb were raised in isolation (with normal OCB pattern) in $16 / 35$ (46\%) and 24/34 (71\%) patients, respectively. This additional information, although identified in both groups, is clinically relevant. Because systemically derived albumin gets into the CSF through passive diffusion and cannot be produced in the CNS, the $\mathrm{Q}_{\mathrm{Alb}}$ is a useful method to assess the blood-CSF barrier and, when raised, indicates barrier impairment. ${ }^{9}$ In contrast, raised Ig levels in the CSF may be secondary to either bloodbrain barrier disruption or intrathecal synthesis; therefore, the $\operatorname{IgG}$ index is a more sensitive measure of focal IgG production as it adjusts for IgG leakage due to barrier dysfunction and serum albumin and IgG concentration. ${ }^{69}$ Quantitative analysis may also be useful when there is significant damage or impairment to the blood-brain barrier and the OCB patterns may be masked by the background of other proteins.

It is important to acknowledge that our cohort has inherent biases. As this was a pragmatic clinical study, the higher representation of some of the immunemediated conditions may simply reflect the lengths physicians go to investigate them, in contrast to conditions where the clinical diagnosis is based on good diagnostic criteria, such as MS, or a disease biomarker, such as the autoimmune encephalitides. Sometimes patients with incomplete investigations performed prior to referral would not have a repeat CSF analysis and were subsequently excluded from the study. Thus, our study may not reliably evaluate for specific disorders, such as MS, that are more susceptible to these influencing factors. Nevertheless, both quantitative and qualitative analyses of CSF are useful in identifying the group of inflammatory disorders.

The early neurologic features of inflammatory disorders can be indistinguishable from a range of other "noninflammatory" disorders; CSF analysis would have been performed in patients who are subsequently diagnosed with a range of these conditions, as in reflected in our cohort. However, this has provided important information on the possible inflammatory mechanisms in these disorders. In our study, both qualitative and quantitative abnormalities were seen in patients with noninflammatory disorders, in keeping with previous reports of immune and inflammatory mechanisms being implicated in a broad range of neurodegenerative disorders ${ }^{10}$ and epilepsies. ${ }^{11}$ Hence, when evaluating the diagnostic accuracy of such an analysis that identifies an inflammatory mechanism, care needs to be taken when CNS disorders are classified only according to their primary pathology.

Taken together, our data support the use of OCB analysis in pediatric inflammatory neurologic conditions, as has been previously reported in other pediatric and adult cohorts. However, both the IgG index and $\mathrm{Q}_{\text {Alb }}$ can be additionally informative and should be performed as a standard evaluation as they may also have utility in monitoring inflammatory mechanisms and therapy response. Further studies should evaluate the additional utility of these biomarkers, either individually or in combination with other markers of inflammation such as CSF reactivity, imaging, and CSF neopterins, ${ }^{12}$ in evaluating inflammatory conditions and/or mechanisms.

\section{AUTHOR CONTRIBUTIONS}

Yael Hacohen: drafting/revising the manuscript, study concept or design, analysis or interpretation of data, acquisition of data. Rahul Singh: acquisition of data, drafting/revising the manuscript. Vhari Forsyth: acquisition 
of data, drafting/revising the manuscript. Michael Absoud: drafting/revising the manuscript, study concept or design, statistical analysis, analysis or interpretation of data. Ming Lim: drafting/revising the manuscript, study concept or design, study supervision.

\section{STUDY FUNDING}

This work was supported by the National Institute for Health Research (NIHR) Oxford Biomedical Research Centre based at Oxford University Hospitals NHS Trust and the University of Oxford.

\section{DISCLOSURE}

Y. Hacohen, R. Singh, and V. Forsyth report no disclosures relevant to the manuscript. M. Absoud has received funding from the MS Society and Action Medical Research charities and is on the editorial advisory board for the International Journal of Language \& Communication Disorders. M. Lim receives research grants from Action Medical Research, SPARKS, Great Ormond Street Hospital Charity, DES charities, and MS Society; receives research support grants from the London Clinical Research Network and Evelina Appeal; has received consultation fees from CSL Behring; received travel grants from Merck Serono; and was awarded educational grants to organize meetings by Novartis, Biogen Idec, Merck Serono, and Bayer. Go to Neurology.org/nn for full disclosures.

Received February 27, 2014. Accepted in final form March 31, 2014.

\section{REFERENCES}

1. Freedman MS, Thompson EJ, Deisenhammer F, et al. Recommended standard of cerebrospinal fluid analysis in the diagnosis of multiple sclerosis: a consensus statement. Arch Neurol 2005;62:865-870.

2. Zeman A, McLean B, Keir G, Luxton R, Sharief M, Thompson E. The significance of serum oligoclonal bands in neurological diseases. J Neurol Neurosurg Psychiatry 1993;56:32-35.

3. Kostulas V, Eeg-Olofsson O, Olsson T, Link H. Demonstration in children of oligoclonal $\mathrm{IgG}$ bands in unconcentrated
CSF using agarose isoelectric focusing and immunolabeling. Pediatr Neurol 1986;2:286-289.

4. Sinclair A, Wienholt L, Tantsis E, Brilot F, Dale RC. Clinical association of intrathecal and mirrored oligoclonal bands in paediatric neurology. Dev Med Child Neurol 2013;55: 71-75.

5. Reiber H, Peter JB. Cerebrospinal fluid analysis: disease-related data patterns and evaluation programs. J Neurol Sci 2001;184: 101-122.

6. Reiber H, Thompson EJ, Grimsley G, et al. Quality assurance for cerebrospinal fluid protein analysis: international consensus by an Internet-based group discussion. Clin Chem Lab Med 2003;41:331-337.

7. Krupp LB, Tardieu M, Amato MP, et al. International Pediatric Multiple Sclerosis Study Group criteria for pediatric multiple sclerosis and immune-mediated central nervous system demyelinating disorders: revisions to the 2007 definitions. Mult Scler 2013;19:1261-1267.

8. Hacohen $Y$, Wright $S$, Waters $P$, et al. Paediatric autoimmune encephalopathies: clinical features, laboratory investigations and outcomes in patients with or without antibodies to known central nervous system autoantigens. J Neurol Neurosurg Psychiatry 2013;84:748-755.

9. King MD, Stephenson JBP. A Handbook of Neurological Investigations in Childhood, 1st ed. London: Mac Keith Press; 2009;2.7:93-94.

10. Lim M. Treating inflammation in childhood neurodegenerative disorders. Dev Med Child Neurol 2011;53: 298-304.

11. Vezzani A, Friedman A. Brain inflammation as a biomarker in epilepsy. Biomark Med 2011;5:607-614.

12. Dale RC, Brilot F. Biomarkers of inflammatory and autoimmune central nervous system disorders. Curr Opin Pediatr 2010;22:718-725. 


\title{
Neurology \\ Neuroimmunology \& Neuroinflammation
}

\author{
CSF albumin and immunoglobulin analyses in childhood neurologic disorders \\ Yael Hacohen, Rahul Singh, Vhari Forsyth, et al. \\ Neurol Neuroimmunol Neuroinflamm 2014;1; \\ DOI 10.1212/NXI.0000000000000010
}

This information is current as of May 15, 2014

\section{Updated Information \& Services}

References

Subspecialty Collections

Permissions \& Licensing

\section{Reprints}

including high resolution figures, can be found at:

http://nn.neurology.org/content/1/1/e10.full.html

This article cites 12 articles, 2 of which you can access for free at: http://nn.neurology.org/content/1/1/e10.full.html\#\#ref-list-1

This article, along with others on similar topics, appears in the following collection(s):

All Immunology

http://nn.neurology.org//cgi/collection/all_immunology

All Pediatric

http://nn.neurology.org//cgi/collection/all_pediatric

Information about reproducing this article in parts (figures,tables) or in its entirety can be found online at:

http://nn.neurology.org/misc/about.xhtml\#permissions

Information about ordering reprints can be found online:

http://nn.neurology.org/misc/addir.xhtml\#reprintsus

Neurol Neuroimmunol Neuroinflamm is an official journal of the American Academy of Neurology.

Published since April 2014, it is an open-access, online-only, continuous publication journal. Copyright $\odot$ 2014 American Academy of Neurology. All rights reserved. Online ISSN: 2332-7812.

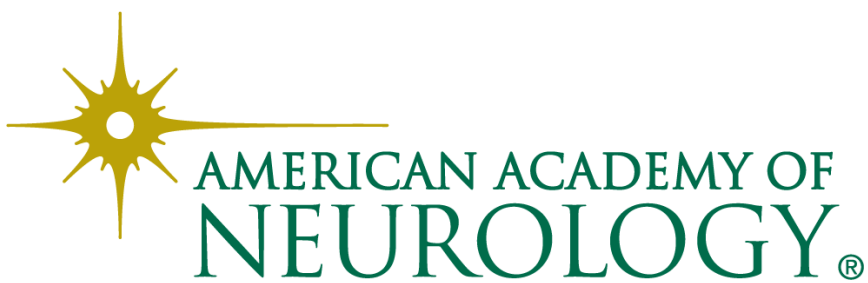

